\title{
Differential proteomic analysis of pathway biomarkers in human breast cancer by integrated bioinformatics
}

\author{
LIU FU-JUN $^{1 *}$, JIN SHAO-HUA $^{1 *}$ and SHEN XIAO-FANG ${ }^{2}$ \\ ${ }^{1}$ Central Laboratory; ${ }^{2}$ Reproduction Medical Center, Yu-Huang-Ding Hospital/Qingdao University, \\ Yantai, Shandong 264000, P.R. China
}

Received March 28, 2012; Accepted July 24, 2012

DOI: $10.3892 / \mathrm{ol} .2012 .881$

\begin{abstract}
The aim of this study was to better understand the altered functional modules in breast cancer at pathway and network levels. An integrated bioinformatics analysis of differentially expressed proteins in human breast cancer was performed. Breast cancer protein profiles were constructed by data mining proteins in literature and public databases, including 1031 proteins with 153 secretory and 69 cell surface proteins. An experimental investigation was performed by two-dimensional electrophoresis, and 4 proteins were further validated by western blotting. Enriched bioinformatics functions were clustered. This study may be used as a reference in further studies to help identify the underlying biological interactions associated with breast cancer and discover potential cancer targets.
\end{abstract}

\section{Introduction}

Breast cancer is the most common neoplasia in women and its pathogenesis is related to an acquired or inherited genetic disorder influenced by environmental, behavioral or reproductive factors (1). Cancer biomarker discovery is important for both cancer biology and clinical applications. These markers may come from DNA, RNA, miRNA or proteins (2), with proteins being the most significant (3).

The development and improvement of biotechnologies has allowed researchers to perform high-throughput analyses of genomes, transcriptomes and proteomes in health and disease, and identify hundreds of potential biomarkers (4), offering the potential to discover diagnostic, prognostic or therapeutic targets. However, less than two dozen cancer biomarkers are

Correspondence to: Professor Shen Xiao-Fang, Reproduction Medical Center, Yu-Huang-Ding Hospital, 20 YuDong Road, Yantai, Shandong 264000, P.R. China

E-mail: sdstemcell@126.com

*Contributed equally

Key words: bioinformatics, biomarker, breast cancer, cancer/testis antigen, proteomes currently approved by the Food and Drug Administration (FDA) (5), including only 9 protein biomarkers identified in the blood (6). Due to the lack of sensitivity and specificity of these known biomarkers (7), researchers continue to search for more significant targets. Proteomics is a promising approach for the discovery of cancer targets and biomarkers (8). The mapping of proteome profiles and differential proteomics has been widely performed in breast cancer to identify potential biomarkers (9). The identified proteins were reported to have potential clinical significance, and certain proteins may be used as potential diagnostic, prognostic or predictive biomarkers $(10,11,12,13,14)$. However, due to the heterogeneity in the different studies, including experimental design, sample collection and classification and analytical method (15), these results lack good reproducibility and require further validation before they can be used in clinical detection and to explain the underlying mechanisms of breast cancer. In addition, few protein candidates were warranted to be specific to breast cancer, and were often differentially expressed in other cancer types (16). Hence, research encountered the challenge of how to decipher and use these individual results and bring them into clinical applications. In addition, an understanding of the underlying biological mechanisms of carcinogenesis and the altered molecular events in breast cancer at integrated pathway levels is necessary. Proteins do not act alone to perform biological functions, but through complex biological pathways. The discovery of these intricate pathways is essential to understanding biological mechanisms. A wide variety of cancers may also link to the same pathways that affect tumorigenesis and progression through altering protein expressions. Therefore, once these pathways are known, it may be easier to monitor different aspects of cancer progression and develop a therapeutic strategy by focusing on pathways instead of individual proteins. The enriched pathways or functions may be the most probable cause of cancer (17), and the enriched proteins involved in these processes could in turn serve as target agents in diagnosis or treatment. Several monoclonal antibodies and small molecular inhibitors have been developed to target certain molecular pathways involved in cell growth, survival and metastasis in breast cancer $(18,19,20)$. Therefore, integrated bioinformatics should be applied when discovering cancer-associated pathways and networks to improve our understanding of cancer biology, as well as cancer diagnosis and therapeutics. 
In the present study, we integrated protein profiles in breast cancers from literature and public databases to perform bioinformatical analyses. Differential serum proteomics between human breast cancer patients and healthy volunteers were performed, and western blotting was used to validate the differential expression of secretory proteins in breast cancer. Our aim was not to discover all the breast cancer-associated proteins but rather to focus on screening enriched signaling pathways in breast cancer which may provide new insights into breast cancer research. This bioinformatical insight into breast cancerassociated protein profiles may potentially provide clues for identifying new functional modules in breast cancer and may be used to understand the underlying tumorigenesis process.

\section{Materials and methods}

Patient characteristics and serum collection. Blood samples were collected from 25 breast cancer patients and 20 healthy volunteers at the Yantai Yu-Huang-Ding Hospital, China, with written informed consent and approval of the Yu-Huang-Ding Hospital research and ethics committee. Venous blood was drawn from each subject into 10-ml fasting blood tubes and allowed to clot at room temperature for $1 \mathrm{~h}$. Serum was separated by centrifugation at $2000 \mathrm{~g}$ for $15 \mathrm{~min}$ at $4^{\circ} \mathrm{C}$. Proteins in the supernatant were precipitated by mixing with 4 volumes of ice-cold acetone and allowing it to stand at $-20^{\circ} \mathrm{C}$ for $1 \mathrm{~h}$. After being centrifuged at $12000 \mathrm{~g}$ for $1 \mathrm{~h}$, washed with $90 \%$ acetone and dried, the proteins were taken up in $2 \mathrm{ml}$ lysis solution (7 M urea, $2 \mathrm{M}$ thiourea and $65 \mathrm{mM}$ dithiothreitol) and stored at $-80^{\circ} \mathrm{C}$.

Collection of breast cancer-associated proteins. Breast cancer protein profiles were constructed by retrieving public databases (Uniprot, Release 2011_11, http://www.uniprot. org) and literature on differential proteomes in breast cancer (dbDEPC 2.0, http://lifecenter.sgst.cn/dbdepc/index.do). All human proteins in the Uniprot database were downloaded with all annotations. Breast cancer-associated proteins were further selected manually from the downloaded data using the keywords 'cancer', 'tumor' or 'carcinoma'. The regulation levels were recorded as 'over-regulation', 'downregulation' and 'no-annotation'. All proteins extracted from the literature were further grouped into over- and downregulation clusters, and certain proteins with controversial expression in different reports were clustered into the no-annotation group.

\section{Bioinformatics analysis}

Ontological analysis. All breast cancer-associated proteins were classified into different protein classes according to Interpro (www.ebi.ac.uk/interpro) and Gene Ontology (GO) annotation (www.geneontology.org), and significantly enriched functions were further selected according to Panther (http://www.pantherdb.org/).

Pathway analysis. Ingenuity Pathway Analysis v8.0-2803 (Ingenuity Systems, Redwood City, CA, USA) was used to analyze pathways and networks involving the breast cancer-associated proteins. The following settings were used: reference set, Ingenuity Knowledge Base (genes only); network analysis, direct and indirect relationships; molecules per network, 35; networks per analysis, 25. All species, tissues and cell lines were used for the analysis. IPA uses Fisher's exact test to determine which pathways (canonical pathways, toxicity pathways or biological functions) are significantly linked to the input protein set compared with the whole Ingenuity Knowledge Base.

Selection of secretory and cell surface proteins. Secretory proteins and cell surface proteins are promising biomarkers. All cancer-associated proteins were compared with the serum/plasma proteome (21) to select secretory proteins, and compared with the cell surfaceome (22) to select cell surface proteins. GO was used to further filter the results.

Proteomic analysis of human serum from patients with breast cancer and healthy volunteers. Two-dimensional gel electrophoresis and mass spectrometric analyses were performed as described in our previous study (23). Gels were made in triplicate to confirm the spot patterns and were scanned with a Z320 scanner (Founder, Beijing, China). The gel images were processed with ImageMaster software (GE Healthcare, Piscataway, NJ, USA). Images were briefly checked manually to eliminate artefacts. Following spot detection, a match set was built including all the experimental and control gels. The significance of the differential expression of protein spots between experimental and control groups was estimated by mean ratio $>2.0$ and the independent samples t-test; $\mathrm{P}<0.05$ was considered to indicate a statistically significant result.

Western blot analysis. A total of $50 \mu \mathrm{g}$ of pooled proteins were separated at $12 \%$ SDS-PAGE by electrophoresis and then transferred onto a nitrocellulose membrane (Millipore, Bedford, MA, USA). The membranes were blocked with $5 \%$ milk TBST (10 mM Tris- $\mathrm{HCl}, \mathrm{pH} 8.0,150 \mathrm{mM} \mathrm{NaCl}$, $0.1 \%$ Tween-20) overnight at $4{ }^{\circ} \mathrm{C}$ and then hybridized with the following primary antibodies: anti-ORM2 (sc-51020), anti-APOA1 (sc-69755), anti-GC (sc-18706) and anti-CLU (sc-166907), all from Santa Cruz Biotechnology, Inc. (Santa Cruz, CA, USA). The immune complexes were visualized with a DAB staining kit (Zhongshan Jinqiao Technology, China).

\section{Results}

Overview of breast cancer-associated proteins. The breast cancer-associated protein profile was mainly comprised of proteins screened from the Uniprot database and literature reports. A total of 803 breast cancer-associated proteins were screened from literature reports and 243 were selected in the Uniprot database. Finally, 1031 breast cancer-associated proteins were obtained including $514(49.6 \%)$ upregulated and 318 $(30.8 \%)$ downregulated proteins. One hundred and seventy-two proteins required further confirmation of expression.

\section{Bioinformatics analysis}

From protein lists to biological functions. According to GO analysis, Interpro and literature annotations, the enriched biological functions are listed in Table I. Metabolic process, chaperone transcription and catalytic activity-associated proteins were more significantly enriched in breast cancerassociated proteins. The comparison of up- and downregulated proteins demonstrated that more chaperone, cell adhesion, transporter and antioxidant-related proteins were present in the upregulated group, while defense/immunity and extracellular proteins were present in the downregulated group. 


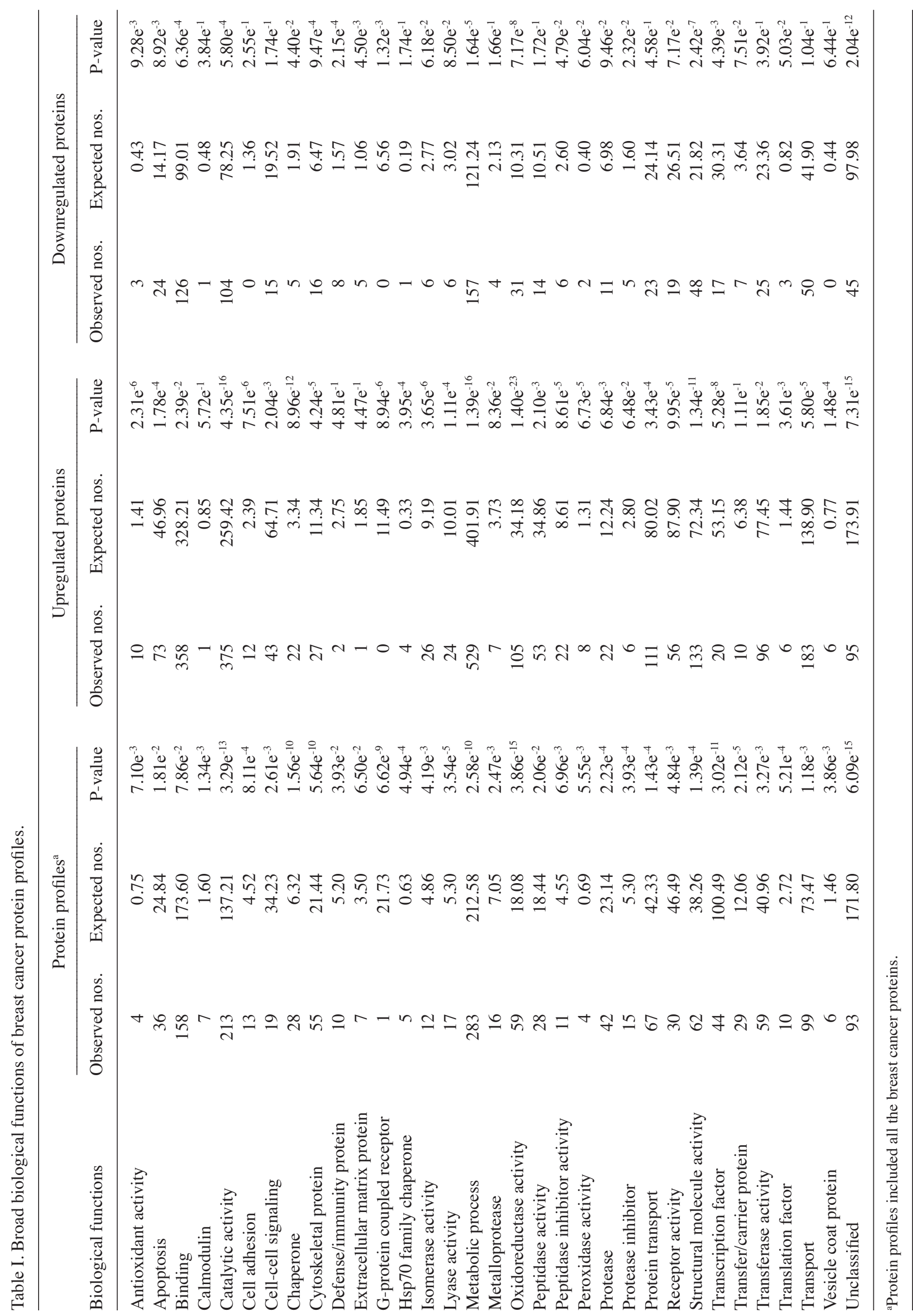


Table II. Canonical pathways in up- and downregulated breast cancer proteins.

\begin{tabular}{|c|c|c|c|}
\hline Pathway & Ratio & Number & P-value \\
\hline \multicolumn{4}{|l|}{ Upregulated } \\
\hline Glycolysis & 0.18 & 15 & $4.88 e^{-8}$ \\
\hline Pyruvate metabolism & 0.18 & 11 & $3.52 \mathrm{e}^{-6}$ \\
\hline Protein ubiquitination pathway & 0.09 & 23 & $1.30 \mathrm{e}^{-5}$ \\
\hline RhoA signaling & 0.12 & 13 & $5.00 \mathrm{e}^{-5}$ \\
\hline NRF2-mediated oxidative stress response & 0.09 & 17 & $6.71 \mathrm{e}^{-5}$ \\
\hline PI3K/AKT signaling & 0.10 & 13 & $8.75 \mathrm{e}^{-5}$ \\
\hline Fatty acid metabolism & 0.13 & 13 & $2.62 \mathrm{e}^{-4}$ \\
\hline Integrin signaling & 0.08 & 16 & $2.80 \mathrm{e}^{-4}$ \\
\hline ILK signaling & 0.08 & 15 & $2.82 \mathrm{e}^{-4}$ \\
\hline 14-3-3-mediated signaling & 0.10 & 12 & $3.10 \mathrm{e}^{-4}$ \\
\hline RAN signaling & 0.28 & 5 & $3.16 \mathrm{e}^{-4}$ \\
\hline Aryl hydrocarbon receptor signaling & 0.12 & 17 & $3.48 \mathrm{e}^{-4}$ \\
\hline Clathrin-mediated endocytosis signaling & 0.08 & 14 & $6.40 \mathrm{e}^{-4}$ \\
\hline Valine, leucine and isoleucine degradation & 0.13 & 8 & $7.71 \mathrm{e}^{-4}$ \\
\hline IGF-1 signaling & 0.10 & 10 & $1.12 \mathrm{e}^{-3}$ \\
\hline VEGF signaling & 0.10 & 9 & $1.75 \mathrm{e}^{-3}$ \\
\hline Arginine and proline metabolism & 0.11 & 8 & $2.10 \mathrm{e}^{-3}$ \\
\hline EIF2 signaling & 0.07 & 14 & $2.19 \mathrm{e}^{-3}$ \\
\hline Actin cytoskeleton signaling & 0.07 & 15 & $2.96 \mathrm{e}^{-3}$ \\
\hline ERK5 signaling & 0.11 & 7 & $3.55 \mathrm{e}^{-3}$ \\
\hline GABA receptor signaling & 0.13 & 6 & $4.00 \mathrm{e}^{-3}$ \\
\hline Regulation of actin-based motility by Rho & 0.09 & 8 & $5.16 \mathrm{e}^{-3}$ \\
\hline LPS/IL-1 mediated inhibition of RXR function & 0.06 & 13 & $9.42 \mathrm{e}^{-3}$ \\
\hline HER-2 signaling in breast cancer & 0.09 & 7 & $1.10 \mathrm{e}^{-2}$ \\
\hline \multicolumn{4}{|l|}{ Downregulated } \\
\hline Citrate cycle & 0.09 & 5 & $1.25 \mathrm{e}^{-4}$ \\
\hline Acute phase response signaling & 0.06 & 10 & $6.53 \mathrm{e}^{-4}$ \\
\hline P53 signaling & 0.07 & 7 & $1.13 \mathrm{e}^{-3}$ \\
\hline Primary immunodeficiency signaling & 0.09 & 5 & $1.80 \mathrm{e}^{-3}$ \\
\hline Urea cycle and metabolism of amino groups & 0.13 & 4 & $2.76 \mathrm{e}^{-3}$ \\
\hline Cdc42 signaling & 0.06 & 8 & $3.40 \mathrm{e}^{-3}$ \\
\hline Glyoxylate and dicarboxylate metabolism & 0.18 & 4 & $1.12 \mathrm{e}^{-3}$ \\
\hline Autoimmune thyroid disease signaling & 0.08 & 3 & $1.02 \mathrm{e}^{-3}$ \\
\hline
\end{tabular}

From biological functions to pathways. To explore the enrichment pathways of these proteins with different biological functions, a pathway analysis was performed using Ingenuity Pathway Analysis tools and the Kyoto Encyclopedia of Genes and Genomes (KEGG) database. Thirty-three pathways were identified in breast cancers, including 25 pathways enriched in upregulated proteins and 8 pathways with downregulated proteins (Table II).

From biological pathways to networks. Network generation was performed using Ingenuity Pathway Analysis tools. This generated 25 networks using all the breast cancer-associated proteins and 13 networks using the upregulated proteins. Several functions were linked to more than 3 of the networks in the upregulated group and the main ones included cancer, genetic disorder, cell death and cellular movement.
Cancer/testis proteins, secretory proteins and cell surface proteins. Cancer/testis antigens are potential cancer biomarkers with restricted expression in the testis and certain cancer cells. The earlier comparison of breast cancer proteins showed that 119 testis proteins including 11 testis-specific proteins were included in this study. Twenty-one proteins were common to both the breast cancer proteins and cancer/testis antigens. The comparison of breast cancer proteins with human serum/plasma and cell surface proteins (surfaceome) revealed that the cancer proteins included 153 secretory proteins and 69 cell surface proteins

Experimental analysis by $2 D$-electrophoresis and western blotting. All gels were statistically analyzed through ImageMaster software, and 11 proteins were shown to be 


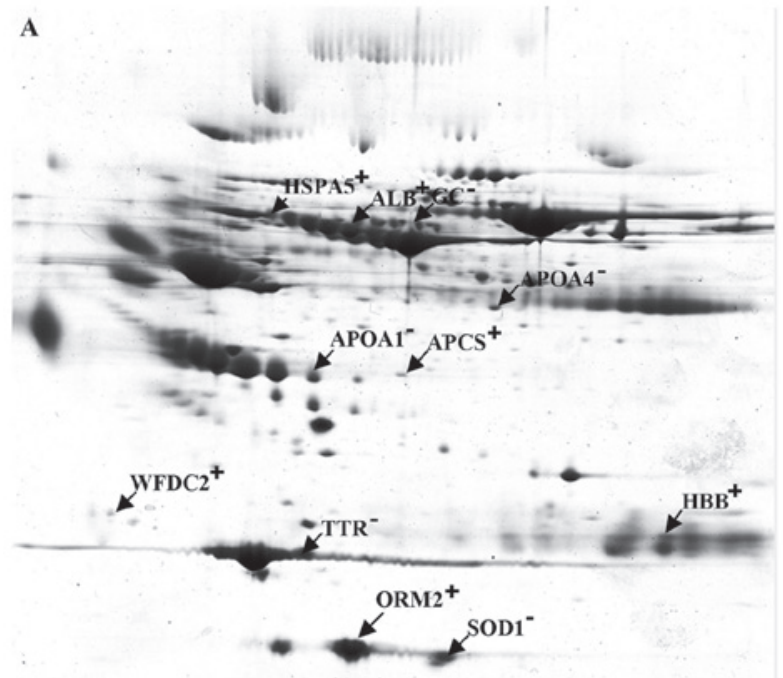

B

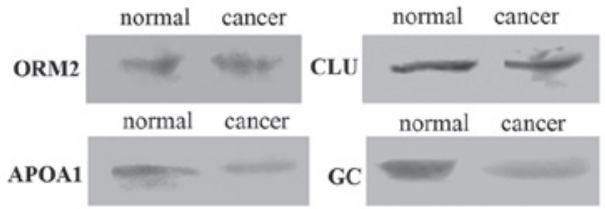

C

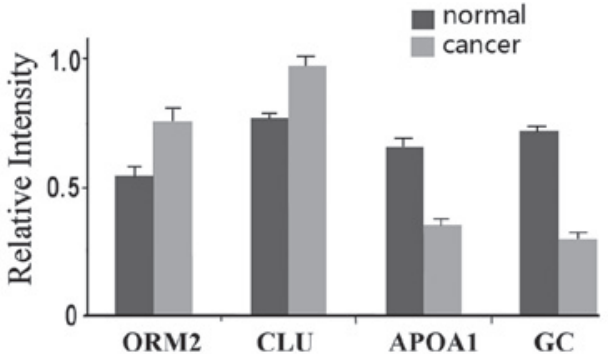

Figure 1. Western blot analysis of ORM2, CLU, APOA1 and GC. (A) Separation of breast cancer serum proteins by 2D-PAGE; (B) Western blot analysis was performed for the serum of breast cancer patients and healthy volunteers; (C) Western blot images were quantified by densitometric scanning and quantified using ImageQuant software.

differentially expressed between breast cancer patients and healthy donors (Fig. 1A). Of the 4 proteins tested by western blotting, ORM2 and CLU were verified to be highly expressed in the serum of breast cancer patients, and APOA1 and GC were expressed at low levels (Fig. 1B).

\section{Discussion}

With the development and improvement of proteomic technology, cancer proteomes are evolving quickly and aid in the search for biomarkers and therapeutic targets. Breast cancer proteomes were widely analyzed and certain important proteins were identified $(9,10)$. However, these data were heterogeneous and many of them lacked experimental verification and validation. In our preliminary study, 11 serum proteins were found to be differentially expressed between breast cancer patients and healthy individuals. The results provided insight into certain aspects of breast cancer, but had little correlation with all the breast cancer proteomes available. Thus, the integration of protein lists containing breast cancer proteomes to understand the correlations involved in regulation functions and networks may aid in the screening of diagnostic markers or therapeutic targets.

In the present study, integrated bioinformatical tools were used to analyze the enriched pathways and networks that are associated with breast cancer. The breast cancer protein profile was constructed, including proteins in the published literature of differential proteomics and the Uniprot database. Protein expressions in breast cancer were evaluated, and broadly categorized into three groups: upregulated, downregulated and those that had contradictory expressions in breast cancer. An ontological analysis indicated that most of these breast cancer-associated proteins were classified into different enrichment functional groups that may be involved in different aspects of cancer biology. The pathway networks analysis showed that several signaling pathways and networks were significantly associated with human breast cancer. These pathways and functions were relevant to the cancer microenvironment, invasion and metastasis processes as briefly described below.

Previous studies have shown that the cancer microenvironment is deficient in oxygen, low in glucose (24), and is is usually followed by highl glycolytic activity $(25,26)$. Studies of breast cancers showed that hypoxic conditions may alter the expression of certain proteins that serve as hypoxic markers (27). Fifteen glycolytic enzymes were investigated in the present study which may be involved in tumor proliferation in hypoxic conditions. The protein ubiquitination pathway and the PI3K/Akt signaling pathway have been reported to be associated with the development of human breast cancers $(28,29)$. Certain drugs have been applied to affect these pathways in the treatment of breast cancer $(30,31,32,33)$. The RhoA signaling pathway was strongly correlated with tumor cell invasion and metastasis (34), and the Nrf2-mediated oxidative stress response activated genes encoding detoxification enzymes and antioxidant proteins to protect cells from oxidative stress. In breast cancer cells, NRP/B was able to enhance oxidative stress responses via the Nrf2 pathway (35). More than 13 cell adhesion proteins in the present study may play roles in the intercellular and cell-extracellular matrix interactions of cancer, leading to cancer invasion or metastasis (36), or participating in signal transduction, cell growth and differentiation (37). E-cadherin is one prominent adhesion molecule that forms the E-cadherin-catenin complex which plays a role in epithelial cell-cell adhesion and differentiation (38), in particular serving as a potent invasion/tumor suppressor of breast cancer (39). Previous studies indicated that the downregulation of E-cadherin was relevant to several pathways including the integrin-linked kinase (ILK) signaling pathway $(40,41)$. The ILK signaling pathway was one prominent enrichment pathway in breast cancer, and may play an important role in hormonal cancer progression (42). 
Some of these canonical pathways are known to participate in cancer processes, but the mechanism and the altered individual members are not well studied. We hypothesized that the altered expressions of different members or activators in these enriched pathways may be associated with the tumor process and its microenvironment alteration, and may serve as important targets or agents in the diagnosis and treatment of breast cancer.

More than 200 cancer/testis antigens have been listed in the cancer/testis database (http://www.cta.lncc.br/). The significant feature of these proteins is their restricted expression in the testis and low or no expression in normal tissues (43); therefore, they may be used as potential cancer vaccine targets. Twenty-one cancer/testis antigens (8\%) and 119 testis proteins (11 testis-specific proteins) were included in the breast cancer protein profiles and these proteins warrant further study. Among these proteins, a recent study showed that MAGE-A3 and A4 in the peripheral blood of breast cancer patients may have potential prognostic and predictive implications (44). All of these proteins are promising specific tumor markers of breast cancer.

Due to the limitations of protein identification using current proteomic techniques, some molecules in certain pathways may be missing. However, the present study gives a new bioinformatical insight into breast cancer at systems biology levels by integrating the individual studies to identify enriched biological and molecular pathways, providing vital evidence for future research. Further studies are warranted to substantiate the enriched functions and pathways. The studies may advance our understanding of cancer biomarker discovery, and also facilitate the biological interpretation of cancer biology in a network context.

\section{References}

1. Warner E: Clinical practice. Breast-cancer screening. N Engl J Med 365: 1025-1032, 2011.

2. Aggarwal C, Somaiah N and Simon GR: Biomarkers with predictive and prognostic function in non-small cell lung cancer: ready for prime time? J Natl Compr Canc Netw 8: 822-832, 2010.

3. Tan Y, Ma SY, Wang FQ, Meng HP, Mei C, Liu A and Wu HR: Proteomic-based analysis for identification of potential serum biomarkers in gallbladder cancer. Oncol Rep 26: 853-859, 2011.

4. Abu-Asab MS, Chaouchi M, Alesci S, Galli S, Laassri M, Cheema AK, Atouf F, VanMeter J and Amri H: Biomarkers in the age of omics: time for a systems biology approach. OMICS 15: 105-112, 2011.

5. Dunn BK, Wagner PD, Anderson D and Greenwald P: Molecular markers for early detection. Semin Oncol 37: 224-242, 2010.

6. Ludwig JA and Weinstein JN: Biomarkers in cancer staging, prognosis and treatment selection. Nat Rev Cancer 5: 845-856, 2005 .

7. Wistuba II, Gelovani JG, Jacoby JJ, Davis SE and Herbst RS: Methodological and practical challenges for personalized cancer therapies. Nat Rev Clin Oncol 8: 135-141, 2011.

8. Schmitz-Spanke S and Rettenmeier AW: Protein expression profiling in chemical carcinogenesis: a proteomic-based approach. Proteomics 11: 644-656, 2011.

9. Lau TY, O'Connor DP, Brennan DJ, Duffy MJ, Pennington SR and Gallagher WM: Breast cancer proteomics: clinical perspectives. Expert Opin Biol Ther 7: 209-219, 2007.

10. Hondermarck H, Tastet C, El Yazidi-Belkoura I, Toillon RA and Le Bourhis X: Proteomics of breast cancer: the quest for markers and therapeutic targets. J Proteome Res 7: 1403-1411, 2008.

11. Böhm D, Keller K, Wehrwein N, Lebrecht A, Schmidt M, Kölbl H and Grus FH: Serum proteome profiling of primary breast cancer indicates a specific biomarker profile. Oncol Rep 26: 1051-1056, 2011 .
12. Kadowaki M, Sangai T, Nagashima T, Sakakibara M, Yoshitomi H, Takano S, Sogawa K, Umemura H, Fushimi K, Nakatani Y, Nomura F and Miyazaki M: Identification of vitronectin as a novel serum marker for early breast cancer detection using a new proteomic approach. J Cancer Res Clin Oncol 137: 1105-1115, 2011.

13. Balluff B, Elsner M, Kowarsch A, Rauser S, Meding S, Schuhmacher C, Feith M, Herrmann K, Röcken C, Schmid RM, Höfler H, Walch A and Ebert MP: Classification of HER2/neu status in gastric cancer using a breast-cancer derived proteome classifier. J Proteome Res 9: 6317-6322, 2010.

14. Lai TC, Chou HC, Chen YW, Lee TR, Chan HT, Shen HH, Lee WT, Lin ST, Lu YC, Wu CL and Chan HL: Secretomic and proteomic analysis of potential breast cancer markers by twodimensional differential gel electrophoresis. J Proteome Res 9: 1302-1322, 2010.

15. Issaq HJ, Waybright TJ and Veenstra TD: Cancer biomarker discovery: Opportunities and pitfalls in analytical methods. Electrophoresis 32: 967-975, 2011.

16. Weigelt B, Pusztai L, Ashworth A and Reis-Filho JS: Challenges translating breast cancer gene signatures into the clinic. Nat Rev Clin Oncol 9: 58-64, 2011.

17. Sawyers CL: The cancer biomarker problem. Nature 452: 548-552, 2008

18. Schlotter CM, Vogt U, Allgayer H and Brandt B: Molecular targeted therapies for breast cancer treatment. Breast Cancer Res 10: $211,2008$.

19. Mukai H: Targeted therapy in breast cancer: current status and future directions. Jpn J Clin Oncol 40: 711-716, 2010.

20. Bernard-Marty C, Lebrun F, Awada A and Piccart MJ: Monoclonal antibody-based targeted therapy in breast cancer: current status and future directions. Drugs 66:1577-1591, 2006.

21. Li SJ, Peng M, Li H, Liu BS, Wang C, Wu JR, Li YX and Zeng R: Sys-BodyFluid, a systematical database for human body fluid proteome research. Nucleic Acids Res 37: D907-D912, 2009.

22. da Cunha JP, Galante PA, de Souza JE, de Souza RF, Carvalho PM, Ohara DT, Moura RP, Oba-Shinja SM, Marie SK, Silva WA Jr, et al: Bioinformatics construction of the human cell surfaceome. Proc Natl Acad Sci 106: 16752-16757, 2009.

23. Li J, Liu F, Wang H, Liu X, Liu J, Li N, Wan F, Wang W, Zhang C, Jin S, Liu J, Zhu P and Liu Y: Systematic mapping and functional analysis of a family of human epididymal secretory spermlocated proteins. Mol Cell Proteomics 9: 2517-2528, 2010.

24. Dvorak HF, Weaver VM, Tlsty TD and Bergers G: Tumor microenvironment and progression. J Surg Oncol 103: 468-474, 2011.

25. Gatenby RA and Gillies RJ: Why do cancers have high aerobic glycolysis? Nat Rev Cancer 4: 891-899, 2004.

26. Koppenol WH, Bounds PL and Dang CV: Otto Warburg's contributions to current concepts of cancer metabolism. Nat Rev Cancer 11: 325-337, 2011.

27. Bando H, Toi M, Kitada K and Koike M: Genes commonly upregulated by hypoxia in human breast cancer cells MCF-7 and MDA-MB-231. Biomed Pharmacother 57: 333-340, 2003.

28. Osaki M, Oshimura M and Ito H: PI3K-Akt pathway: its functions and alterations in human cancer. Apoptosis 9: 667-676, 2004

29. Ghayad SE and Cohen PA: Inhibitors of the PI3K/Akt/mTOR pathway: new hope for breast cancer patients. Recent Pat Anticancer Drug Discov 5: 29-57, 2010.

30. Orlowski RZ and Dees EC: The role of the ubiquitinationproteasome pathway in breast cancer: applying drugs that affect the ubiquitin-proteasome pathway to the therapy of breast cancer Breast Cancer Res 5: 1-7, 2003.

31. Rossi S and Loda M: The role of the ubiquitination-proteasome pathway in breast cancer: use of mouse models for analyzing ubiquitination processes. Breast Cancer Res 5: 16-22, 2003.

32. Lipkowitz S: The role of the ubiquitination-proteasome pathway in breast cancer: ubiquitin mediated degradation of growth factor receptors in the pathogenesis and treatment of cancer. Breast Cancer Res 5: 8-15, 2003.

33. McAuliffe PF, Meric-Bernstam F, Mills GB and Gonzalez-Angulo AM: Deciphering the role of PI3K/Akt/mTOR pathway in breast cancer biology and pathogenesis. Clin Breast Cancer 10: S59-S65, 2010.

34. Struckhoff AP, Rana MK and Worthylake RA: RhoA can lead the way in tumor cell invasion and metastasis. Front Biosci 16: 1915-1926, 2011.

35. Seng S, Avraham HK, Jiang S, Yang S, Sekine M, Kimelman N, Li $\mathrm{H}$ and Avraham S: The nuclear matrix protein, NRP/B, enhances Nrf2-mediated oxidative stress responses in breast cancer cells. Cancer Res 67: 8596-8604, 2007. 
36. Martin TA, Mason MD and Jiang WG: Tight junctions in cancer metastasis. Front Biosci 16: 898-936, 2001.

37. Kim SH, Turnbull J and Guimond S: Extracellular matrix and cell signalling: the dynamic cooperation of integrin, proteoglycan and growth factor receptor. J Endocrinol 209: 139-151, 2011.

38. Wijnhoven BP, Dinjens WN and Pignatelli M: E-cadherincatenin cell-cell adhesion complex and human cancer. Br J Surg 87: 992-1005, 2000.

39. Berx G and Van Roy F: The E-cadherin/catenin complex: an important gatekeeper in breast cancer tumorigenesis and malignant progression. Breast Cancer Res 3: 289-293, 2001.

40. Tan C, Costello P, Sanghera J, Dominguez D, Baulida J, de Herreros AG and Dedhar S: Inhibition of integrin linked kinase (ILK) suppresses beta-catenin-Lef/Tcf-dependent transcription and expression of the E-cadherin repressor, snail, in APC-/- human colon carcinoma cells. Oncogene 20: 133-140, 2001.
41. Bravou V, Klironomos G, Papadaki E, Taraviras S and Varakis J: ILK over-expression in human colon cancer progression correlates with activation of beta-catenin, down-regulation of E-cadherin and activation of the Akt-FKHR pathway. J Pathol 208: 91-99, 2006

42. Cortez V, Nair BC, Chakravarty D and Vadlamudi RK Integrin-linked kinase 1: role in hormonal cancer progression. Front Biosci (Schol Ed) 3: 788-796, 2011.

43. Grigoriadis A, Caballero OL, Hoek KS, da Silva L, Chen YT, Shin SJ, Jungbluth AA, Miller LD, Clouston D, Cebon J, Old LJ, Lakhani SR, Simpson AJ and Neville AM: CT-X antigen expression in human breast cancer. Proc Natl Acad Sci 106: 13493-13498, 2009

44. Hussein YM, Gharib AF, Etewa RL,El-Shal AS,Abdel-Ghany ME and Elsawy WH: The melanoma-associated antigen-A3,-A4 genes: relation to the risk and clinicopathological parameters in breast cancer patients. Mol Cell Biochem 351: 261-268, 2011. 